\title{
Orbital Magnetism from Itinerant Electrons
}

\author{
M.S.S. Brooks, G.H. Lander ${ }^{1}$ and B. Johansson ${ }^{2}$ \\ ${ }^{1}$ Institute for Transuranium Elements, Joint Research Centre, Karlsruhe, Germany \\ 2 Institute of Physics, University of Uppsala, Sweden
}

\begin{abstract}
Our understanding of how the outer $5 f$ electrons behave when actinide atoms are brought together to form a compound has been improved by recent advances in calculating the ground-state properties. The main theoretical predictions have been verified by measuring the momentum dependence of neutrons scattered by the unpaired electrons.
\end{abstract}

Most atoms lose their magnetic moments in the solid state, but there are three classes of elements - the $3 d$ transition metals, the $4 \mathrm{f}$ or rare-earth series and the $5 f$ or actinide series where magnetism is retained in the condensed phase. Much of our understanding of solid state magnetism is drawn from the former two groups, primarily owing to the success of the standard models describing localized and itinerant moments.

\section{The Standard Model for Rare-Earth Magnetism}

The standard model of rare-earth magnetism is based upon the approximation that the $4 \mathrm{f}$ states are essentially the same in the solid as in free atoms. Hund's first and second rules then ensure that the total spin and orbital moments are both maximized. Since the $4 \mathrm{f}$ states are spatially deeply buried within the rare-earth atoms, interaction with the environment is weak and is usually adequately represented by local exchange interactions between $4 \mathrm{f}$ and conduction electrons.

In contrast, for the standard model of $3 d$ magnetism, the $3 d$ states are components of the conduction electron band structure. Although Hund's first rule again favours as large a spin moment as possible, this involves for the solid an increase in electron kinetic energy so the spin moments of most

Mike Brooks and Gerry Lander are Senior Scientists working on actinide research at the Institute for Transuranium Elements, CEC Joint Research Centre, W-7500 Karlsruhe, Germany; Borje Johansson is presently a Professor of Physics at Uppsala University, Sweden.

Dr. M.S.S. Brooks graduated from Sussex University, UK, was a postdoc at the University of Pittsburg, USA, and is a Visiting Professor at the University of Uppsala.

Dr. G.H. Lander was educated at Cambridge University, UK, and became Director of $\mathrm{Ar}$ gonne National Laboratory's neutron spallation source in 1981 before returning to Europe in 1986. He is a Visiting Professor at Keele University, UK.

Professor B. Johansson was educated in Sweden and became a professor at the University of Aarhus, Denmark before taking up his present post in 1981. itinerant magnets are therefore not saturated. Hund's second rule plays no part in the standard model of $3 \mathrm{~d}$ magnetism because, to a good approximation, the magnetic moments are pure spin, the orbital moment being quenched.

\section{Spin-Orbit coupling}

In each of the limiting approximations described above, the magnetic anisotropy is zero. In the case of $4 \mathrm{f}$ states, the complete rotational symmetry of the free atom is preserved, whereas for $3 d$ states there is no interaction between spin and orbital degrees of freedom. A better description of reality is obtained in both cases by adding spin orbit coupling - a relativistic effect arising from the fact that a moving electron feels a magnetic field owing to the charge of the nucleus. In transition metals, the effect of such coupling is to mix a small amount of orbital moment into the total moment, thus indirectly coupling the spin direction to the crystal lattice to produce magnetic anisotropy. Hence, the presence of an orbital magnetic moment tends to be associated, in transition metals, with magnetic anisotropy. In the rare earths, on the other hand, spin momenta $S$ and orbital angular momenta $L$ with large values at ambient temperature couple to the total angular momentum, J. Since orbital and spin degrees of freedom are now coupled, any crystalline electric fields (or nonspherical potentials) are sufficient to reduce the rotational symmetry and are responsible for magnetic anisotropy. In this case, however, the crystalline electric fields reduce the total and orbital moments slightly as they lower the symmetry - a process known as quenching. Thus, in rare earths, magnetic anisotropy accompanies a decrease (however small) in the orbital moment whereas in transition metals it accompanies an increase.

The relative importance of spin-orbit interaction on moving across the Periodic Table is illustrated in Fig. 1 where we have plotted the calculated bandwidths of all the transition metals (d-bandwidths), the rare earths (4f-bandwidths) and the actinides (5f-bandwidths) and the corresponding magnitude of spin-orbit splitting. The $5 \mathrm{f}$-bandwidths of the actinides are less than those for the $3 d$ transition metals whereas the spin-orbit interaction for these heavy elements is very large in comparison. Consequently, it would be expected that the combination of orbital moment and magnetic anisotropy due the spin-orbit interaction should be larger for actinide compounds and calculations have shown this to be the case. For instance, the calculated magnitudes of the orbital contributions to the total moments are surprisingly large for $\mathrm{U}, \mathrm{Np}$ and $\mathrm{Pu}$ compounds, and in many cases are in fact larger than the spin contributions. Furthermore, the calculated magnetic anisotropy energies are two orders of magnitude greater than those of rare earths, and five orders of magnitude greater than those of $3 d$ transition metals.

\section{Diverging descriptions}

The existence of a large orbital (or current) contribution to the moment
Fig. 1 - Bandwidth W and spin-orbit splitting $\triangle_{\text {so }}$ across the $d$ and $f$ series elements.

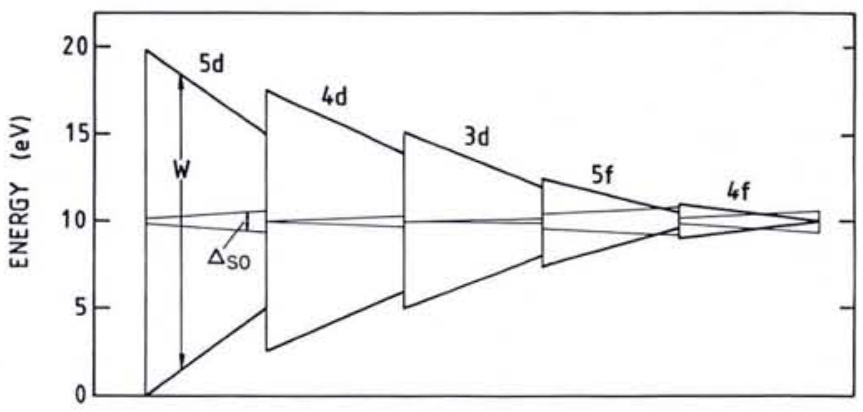

ATOMIC NUMBER 

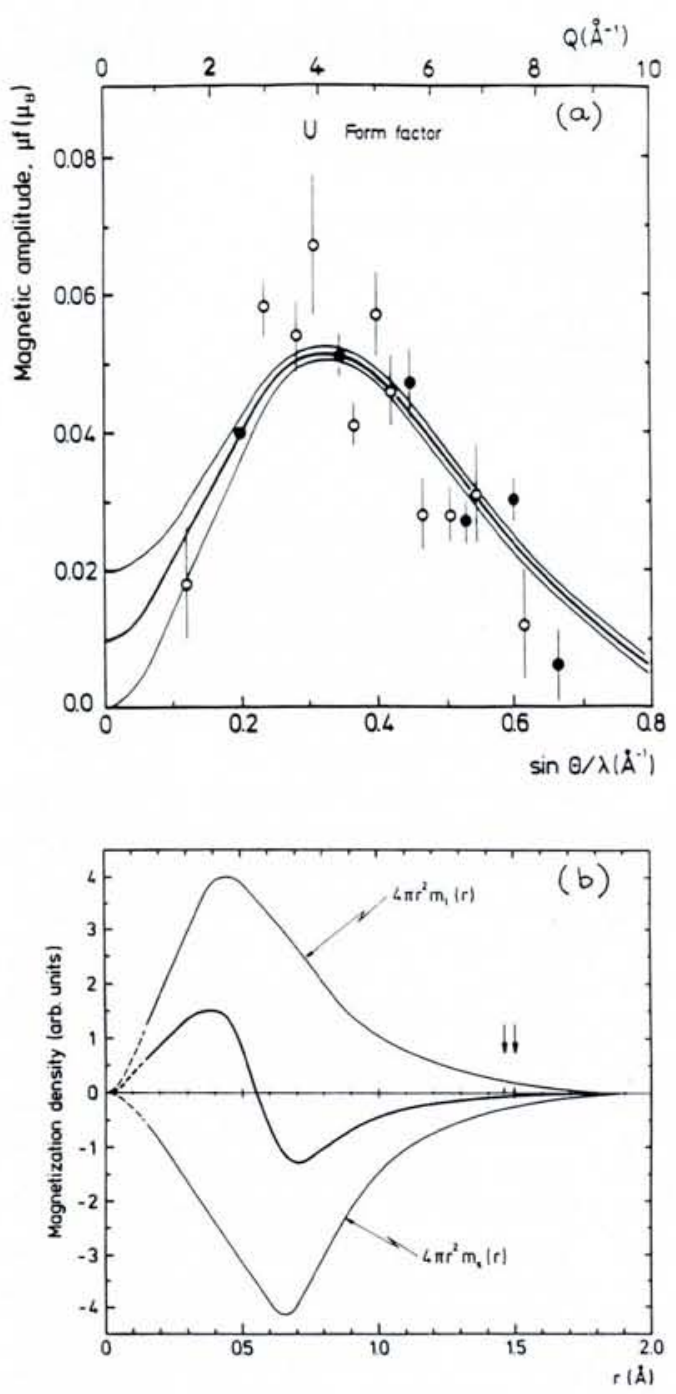

has tended to be associated with localized magnetism on the basis of the standard models described above. This has led to an interesting divergence between descriptions of actinide magnetism proposed by experimentalists and theorists.

For theorists, itinerant magnets normally have only a very small admixture of orbital moment (as described above for the 3d elements) and the orbital moments of itinerant actinide magnets are "giant" by normal standards. Experimentalists have preferred to compare the measured moments in actinides with those of corresponding rare earths since there is, in both cases, a large orbital contribution. Since a greater degree of quenching of the orbital moment arises in actinides, this description emphasizes the reduction of the orbital contribution. These differences arise, of course, because the bases for comparison are two different standard models and they mirror the confusion that may arise, as described above, in associating the magnitude of the orbital moment with magnetic anisotropy.
Fig. 2 - a, upper) The magnitude (in Bohr magnetrons $\mu_{B}$ ) of the product of the moment $\mu$ and the form factor $f$ of the magnetic scattering on the uranium sublattice in $\mathrm{UFe}_{2}$ as a function of the scattering angle. The extrapolation to $\sin \theta / \lambda \approx 0$ gives the total moment for uranium, in this case almost zero. Note the maximum in the scattering cross section for $\sin \theta / \lambda \approx$ $0.3 \AA^{-1}$. The three solid lines are used to indicate the limits $O \leq \mu$ $\leq 0.02 \mu_{B}$ for the extrapolated moment on the uranium sublattice.

b, lower) Schematic representation of the orbital $\left(4 \pi r^{2} m_{1}\right)$ and spin $\left(4 \pi r^{2} m_{s}\right)$ components of the uranium magnetization density in $\mathrm{UFe}^{2}$ as a function of the distance $r$ from the nucleus. The difference (central, bold line) gives the total magnetization density: it clearly has two peaks, one positive and one negative. The integrated areas of the two are the same so that in this case, the total moment is zero. The form factor is the Fourier transform of the bold line; it has a maximum at $Q>0$ owing to the reversal of the sign of the bold line.

\section{Experimental Work}

Theory makes some interesting predictions with respect to the spin and orbital components of the magnetization in actinide compounds. But how are we to observe these effects experimentally? Most techniques are sensitive to the total magnetization, rather than to the individual components, so to separate the spin and orbital components we need to use a scattering technique that is sensitive to the densities of these two components. The scattering of both thermal neutrons and $\mathrm{x}$-rays represent possible methods, but they are not simple experiments.

\section{Neutron scattering}

In the case of neutrons, the scattering in the forward direction is proportional to the total moment (i.e. spin + orbital). However, because the spatial extent of the unpaired electrons is comparable to the neutron wavelength, the scattering cross section decreases as the scattering angle increases. The change of the magnetic scattering cross section as a function of scattering angle is a mea- sure of the magnetization, which is a density, and is called the magnetic form factor $f$. This is normalized such that $f(Q=0)=1$, where the magnitude of the scattering vector $Q=4 \pi \sin \theta / \lambda$ for a reflection with a Bragg angle $\theta$ and radiation with a wavelength $\lambda$. The spin and orbital form factors are different: this is because the neutrons sense the current loop constituting the orbital magnetization, and this density therefore appears more contracted in real space than that due to the spin magnetization. By measuring the form factor, the spin and orbital contributions may be separated, especially when they are in opposite directions and of comparable magnitude.

\section{$X$-ray scattering}

$\mathrm{X}$-rays may also be used to separate the spin and orbital contributions and this in principle represents an easier method because, for certain arrangements of the scattering vector and polarization of the radiation, even the cross section in the forward direction is sensitive to the individual spin and orbital components. However, the magnetic scattering cross section for $\mathrm{x}$-rays is extremely small and experiments are feasible only with the most intense synchrotron sources.

The experiments performed to date have been in the nature of demonstrations on materials in which the spin and orbital moments are known, e.g., Ho metal. In the future, we can anticipate that $\mathrm{x}$-rays will make an important contribution, especially those that will be available at new, high intensity sources such as the European Synchrotron Radiation Facility which is under construction in Grenoble, France.

\section{Results}

The orbital and spin moments as seen by neutron diffraction are presented in Fig. 2a which plots the 5f-magnetic scattering amplitude (i.e. the product of the moment and the form factor) of $\mathrm{UFe}_{2}$ as measured at the Saclay reactor, Paris, by Wulff et al. In an uranium system, the orbital and spin components are in opposite directions (a simple consequence of Hund's 3rd rule at the beginning of the $5 f$ series) since the $f$-shell is less than half filled.

The total $5 f$-moment at the uranium site, which by definition is the magnetic amplitude at $Q=0$, is clearly almost exactly zero in $\mathrm{UFe}_{2}$. However, the positive value (with respect to the iron moment) of the magnetic amplitude at large $Q$ indicates the presence of an orbital magnetization. A detailed analy- 
sis gives the three curves corresponding to the range of possible values $\left(\mu_{\mathrm{u}}\right.$ $=0.01 \mu_{\mathrm{B}}$ and $\left.[0.01 \pm 0.01] \mu_{\mathrm{B}}\right)$ for the ordered total $5 \mathrm{f}$ moment at the uranium site where $\mu_{B}$ is the Bohr magneton. The orbital and spin moments are found to be $0.23(1) \mu_{\mathrm{B}}$ and $-0.22(2) \mu_{\mathrm{B}}$, respectively, and in opposite directions.

The maximum in $f(Q)$ can be physically understood by examining the individual spin and orbital magnetizations, which are plotted in Fig. 2b. The difference between these two densities is given by the central bold line where we have chosen the total moment (the integrated area of the bold curve) to be zero. The scattering amplitude as measured by the neutron experiment is the Fourier transform of the bold curve. The result is zero at $Q=0$, but clearly a positive $f(Q)$ will arise when the Fourier component has a wavelength comparable to the oscillation of the bold line. In fact, a maximum at $Q \approx 4 \AA^{-1}$ is evidently anticipated.

\section{Comparisons with theory}

The experimental results thus show that the orbital $\mu_{\mathrm{L}}$ and spin $\mu_{\mathrm{S}}$ moments almost cancel. The calculated individual values of $\mu_{\mathrm{L}}$ and $\mu_{\mathrm{S}}$ are at present larger than those observed. This is probably connected with the approximations needed to introduce the orbital moments into the bands, and to possible limitations of the local spin density approximation for the exchange and correlation energy. However, the ratio $\mu_{\mathrm{L}} / \mu_{\mathrm{S}}$ is well reproduced by theory. In Fig. 3 we show a plot of $\mu_{\mathrm{L}} / \mu_{\mathrm{S}}$ versus the $f$-electron count for a series of actinide compounds. Almost all these experiments were performed on single crystals at the Institut Laue-Langevin in Grenoble, France, with preliminary experiments being done at Ris $\emptyset$ National Laboratory, Denmark. This plot illustrates a number of points. First, the "transition-metal" picture with itinerant d-electrons, and almost complete quenching of the orbital moments, constitutes the $\mu_{L} \cong 0$ line. Second, the crosses connected by the dashed line are the spectroscopic single-ion plus intermediate coupling values that we would anticipate based on a "rareearth" like approach. Clearly, the results for many actinide ionic compounds, and even semimetals such as USb and PuSb, agree with this approach.

The cases considered here are the intermetallic compounds with strong $\mathrm{f}-\mathrm{d}$ hybridization. The orbital moment is strongly reduced as compared to the localized model, and strongly increased from the value $\left(\mu_{\mathrm{L}} \cong 0\right.$ ) expected if the

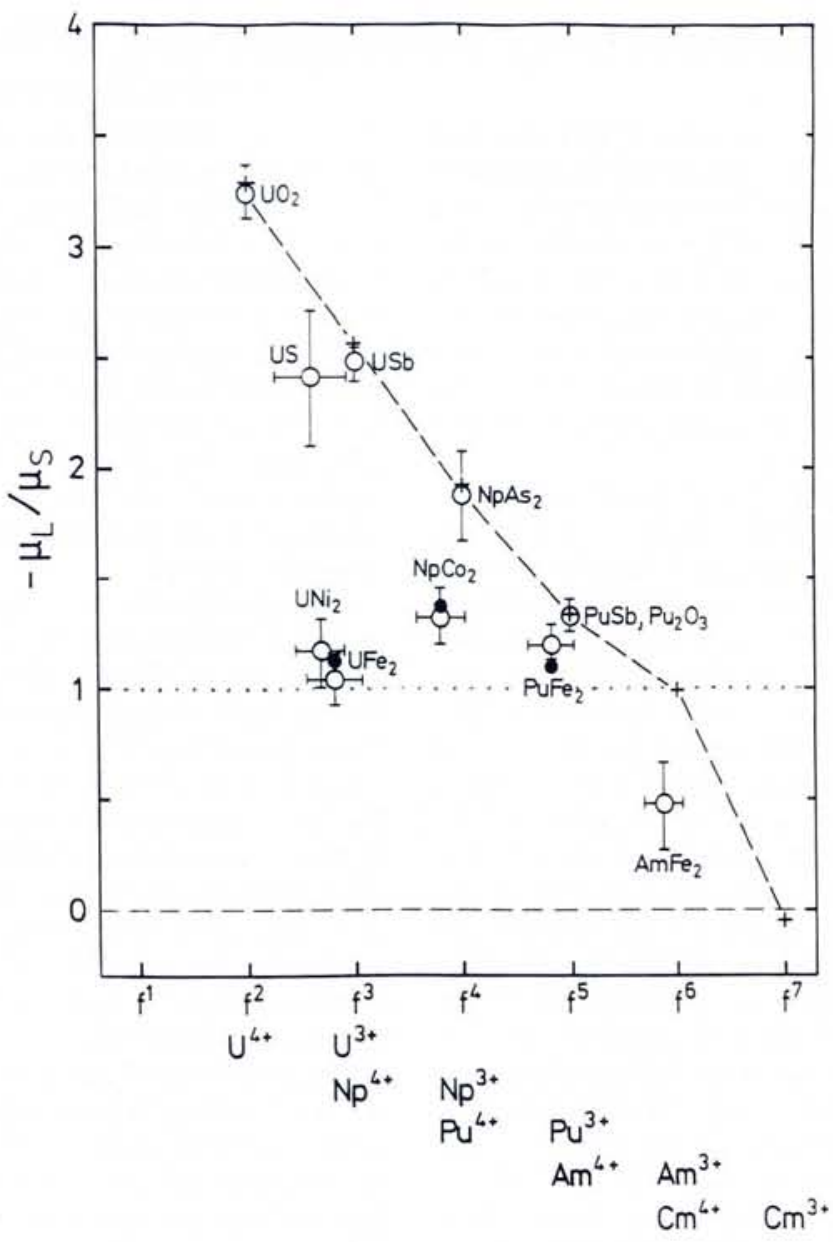

Fig. 3 - The ratio $\mu_{L} / \mu_{S}$ of the orbital and spin moments versus electron count. The crosses are the free-ion $f$ values based on a rare-earth like approach with intermediate coupling. The open points are experimental values and the solid points are the result of the theory. The f-count for the intermetallic compounds is taken from the calculations but is in each case close to that of a tripositive ion.

spin-orbit coupling is not included. The agreement between theory and experiment, at least for the ratio $\mu_{L} / \mu_{S}$, is satisfactory.

\section{$\mathrm{AmFe}_{2}$}

The situation for $\mathrm{AmFe}_{2}$ is particularly intriguing. The experiments were in this case performed at the Argonne National Laboratory many years ago on polycrystalline material. They gave the surprising result that the small moment on the Am site was antiparallel with respect to the Fe moment whereas in all other intermetallics, the actinide moment is found to be parallel to the transition metal moment. We can now understand this long-established result with the aid of Fig. 3. In a localized model on $\mathrm{Am}^{3+}$ there would be zero moment $J$ $=L-S$ and both $L$ and $S$, the orbital and angular spin momenta respectively, are equal to three. However, $\mu_{L}$ is reduced after hybridisation in the compound, allowing $\mu_{\mathrm{s}}$ to dominate and so the moment on $\mathrm{Am}^{3+}$ becomes non-zero. Furthermore, as the absolute value of
$\mu_{\mathrm{S}}$ is larger than $\mu_{\mathrm{L}}$, the moment on $\mathrm{Am}^{3+}$ has the opposite sign to that of actinide moments in other compounds.

In summary, theory and experiment have uncovered a new form of band magnetism in which the orbital moments are large. This has further interesting consequences, some of which, for example the large magnetic anisotropy found in actinide compounds, may even result in practical applications for uranium compounds.

\section{FURTHER READING}

More detailed information is given in: - Theory

Brooks and Kelly, Phys. Rev Lett. 51 (1983) 1708.

Erikson et al., Phys. Rev. B 41 (1990) 9087. - Experiment:

Wulff et al., Phys. Rev. B 37 (1988) 5577; Europhys. Lett. 11 (1990) 269.

Lebech et al., Cond. Matter 1 (1989) 1029. Lander et al., Appl. Phys. Lett. 57 (1990) 989.

Blume and Gibbs, Phys. Rev. B 37 (1988) 1779. 